\title{
5G-XHaul: A Converged Optical and Wireless Solution for 5G Transport Networks
}

Jesús Gutiérrez ${ }^{1 *}$, Nebojsa Maletic ${ }^{1}$, Daniel Camps-Mur ${ }^{2}$, Eduard García ${ }^{2,3}$, Ignacio Berberana ${ }^{4}$ Markos Anastasopoulos ${ }^{5}$, Anna Tzanakaki ${ }^{5}$, Vaia Kalokidou ${ }^{5}$, Paris Flegkas ${ }^{6}$, Dimitris Syrivelis ${ }^{6}$, Thanasis Korakis ${ }^{6}$, Peter Legg ${ }^{7}$, Dusan Markovic ${ }^{8}$, George Limperopoulos ${ }^{9}$, Jens Bartelt ${ }^{10}$, Jay Kant Chaudhary ${ }^{10}$, Michael Grieger ${ }^{11}$, Nikola Vucic ${ }^{12}$, Jim Zou ${ }^{13}$ and Eckhard Grass ${ }^{1,14}$

${ }^{1}$ IHP GmbH, Germany, ${ }^{2}$ i2CAT Foundation, Spain, ${ }^{3}$ Technical University of Catalonia, Spain, ${ }^{4}$ Telefónica I+D, Spain, ${ }^{5}$ University of Bristol, UK, ${ }^{6}$ University of Thessaly, Greece, ${ }^{7}$ Blu Wireless Technology, UK, ${ }^{8}$ TES Electronic Solutions, Germany, ${ }^{9} \mathrm{COSMOTE}$ - Mobile Telecommunications S.A., Greece, ${ }^{10}$ Technical University of Dresden, Germany, ${ }^{11}$ Airrays GmbH, Germany, ${ }^{12} \mathrm{Huawei}$ Technologies Duesseldorf, Germany, ${ }^{13}$ ADVA Optical Networking, Germany, ${ }^{14}$ Humboldt-Universitt zu Berlin, Germany

\begin{abstract}
The common European ICT sector vision for $5 \mathrm{G}$ is that it should leverage on the strengths of both optical and wireless technologies. In the $5 \mathrm{G}$ context, a wide spectra of radio access technologies - such as millimetre wave transmission, massive MIMO, and new waveforms - demand for high capacity, highly flexible and convergent transport networks. As the requirements imposed on future $5 \mathrm{G}$ networks rise, so do the challenges in the transport network. Hence, 5G-XHaul proposes a converged optical and wireless transport network solution with a unified control plane based on software defined networking. This solution is able to support the flexible backhaul and fronthaul - X-Haul - options required to tackle the future challenges imposed by $5 \mathrm{G}$ radio access technologies. $5 \mathrm{G}$-XHaul studies the trade-offs involving fully or partially converged backhaul and fronthaul functions, with the aim of maximising the associated sharing benefits, improving efficiency in resource utilisation, and providing measurable benefits in terms of overall cost, scalability and sustainability. Copyright (c) 2016 John Wiley \& Sons, Ltd.
\end{abstract}

*Correspondence

Jesús Gutiérrez, IHP GmbH, Im Technologiepark 25, 15236, Frankfurt (Oder), Germany.

E-mail: teran@ihp-microelectronics.com

\section{INTRODUCTION}

Future wireless access will extend beyond people, supporting connectivity for any element that may benefit from being connected, thus, facilitating concepts like the Internet of Things (IoT) and machine-to-machine (M2M) communication. The key motivation for $5 \mathrm{G}$ is to provide ubiquitous, high-speed, high-quality wireless broadband coverage to meet societal and industrial needs beyond 2020. To guarantee that networks will be able to cope with the heterogeneous landscape of future services, different fora like the 5G Infrastructure Public Private Partnership (5G-PPP), the ITU Radiocommunication Sector (ITU-R), and the Next Generation Mobile Networks (NGMN) are working on the definition of performance targets for $5 \mathrm{G}$ systems [1].

The considered use cases for 5G [2] [3] are very diverse in terms of capacity and quality of service (QoS) requirements, such as latency, jitter, bit error rate, and device density. This heterogeneity will lead to multiple types of radio access technologies (RATs) coexisting in the context of $5 \mathrm{G}$, and that different types of services will be addressed by different RATs.

The current network architecture uses a relatively monolithic network and transport framework to accommodate a variety of services. The Radio Access Network (RAN) in $5 \mathrm{G}$ will become more heterogeneous and will require flexible topologies and improved transport network performance. Providing a dedicated network for each of these technologies or applications is very complex and not cost effective. A possible solution to this problem would be to use network slicing - a novel architectural concept to support end-to-end (e2e) network slices in the RAN, mobile fronthaul(FH)/backhaul(BH), and core network $(\mathrm{CN})$. In order to support network slicing in the mobile transport network, dynamic network resource management and sharing will be necessary, which requires an integrated mobile transport network, including the mobile FH and $\mathrm{BH}$. 
In view of this, the 5G-PPP project 5G-XHaul aims at developing a transport network able to provide transport services on demand, seen as a generic data forwarding public resource, where every terminal can directly communicate with any other. This involves the development of a suitable flexible and dynamic data plane, based on the integration of optical and wireless network technologies, that is controlled by a unified control plane.

Current transport networks, especially those for $\mathrm{FH}$, have to fulfill stringent requirements in terms of data rates, latency and/or synchronisation. The introduction of new RAN technologies is expected to have a major impact on the transport network, including:

- The optimal functional split of processing functions executed locally at the central unit (CU), or remotely at the Remote Unit (RU) - also known as Baseband unit (BBU) and Remote Radio Head (RRH), respectively - in the Common Public Radio Interface (CPRI). This optimal split represents a key architectural issue, since it relaxes the stringent requirements for transport capacity, delay and synchronisation. The selection of this split depends on the transport network characteristics, the network topology and scale, the type and volume of services to be supported. These functional splits will be further discussed in Section 3.1.1.

- The introduction of higher carrier frequencies in the 5G RAN, such as centimetre wave (cmWave) and millimetre wave (mmWave) in licensed and unlicensed bands which will enable higher data bandwidths and capacity at the expense of higher path losses. The wide bandwidths on the air interface will also require higher capacities in the transport network and potentially an even stricter synchronisation. The increase in the number of antennas, including Massive MIMO, will also require higher capacities in the transport network.

- The plurality of RAN technologies under discussion in the context of 5G. Examples are higher order modulation schemes, new waveforms and technologies for the support of improved latency and resilience, and a large variety of use cases and machine type communications. These new use cases will have to be reflected in the transport network as well, i.e. by providing low latency or high reliability.

In this context, convergence of wireless and optical communications appears to be a strong candidate to fulfill the stringent requirements imposed by the RAN and new RATs. The main goal of the $5 \mathrm{G}-\mathrm{XHaul}$ project is to develop a novel converged optical/wireless architecture and a suitable network management solution for supporting mobile scenarios.

The remainder of this paper is organised as follows. Section 2 describes the different layers of the 5G-XHaul architecture. Section 3 presents the key benefits of the proposed converged transport network along with the enabling technologies, interfaces and control management methods. Section 4 highlights the 5G-XHaul intentions to contribute to standardisation bodies and sketch the expected impact. Finally, the conclusions are presented in Section 5 .

\section{FUNCTIONAL ARCHITECTURE}

5G-XHaul proposes a converged optical/wireless 5G network infrastructure interconnecting computational resources with fixed and mobile users to support both operational network - Cloud RAN (C-RAN) - and end-user computational services, adopting the concept of cloud computing. A layered architecture, inspired by the ETSI Network Function Virtualisation (NFV) standard [4], and the SDN reference architecture [5], are considered to effectively and efficiently provision both end-user and operational services over the proposed infrastructure.

5G-XHaul will adopt a holistic view considering jointly mobile $\mathrm{FH}$ and $\mathrm{BH}$ functions to ensure appropriate allocation of the required resources across all domains. Its objective is twofold: to minimise the operational expenditure of the $\mathrm{FH}$ in terms of power consumption and capital expenditure under the associated strict delay constraints, and to minimise the total e2e service delay in the BH.

Examples of features that enable these benefits include the option to virtualise the separate control plane, using NFV and deploying Virtual Network Functions (VNFs). These are controlled by the SDN controller, to allow on demand resource allocation, able to support dynamically changing workloads [6]. SDN network elements can be treated as VNFs, since they can be implemented as software running on general-purpose platforms in virtualised environments. Both SDN and non-SDN models can be supported by SDN network elements. On the other hand, network applications can include SDN controller functions, or interact with SDN controllers and can themselves provide VNFs. Service Chaining, supporting orchestrated service provisioning over heterogeneous environments, is considered to be one possible network application. Network elements controlled by SDN controllers can provide either VNFs or Physical Network Functions.

Taking advantage of the SDN concept and the benefits of cross-technology virtualisation, 5G-XHaul proposes an overarching layered architecture able to efficiently and effectively support 5G services [7], presented in Figure 1. The 5G-XHaul infrastructure, in accordance to the $5 \mathrm{G}$ vision, exhibits a great degree of heterogeneity in terms of technologies. To address the challenge of managing and operating this type of complex heterogeneous infrastructure in an efficient manner, 5G-XHaul proposes the adoption of SDN and NFV that will be seamlessly integrated. In SDN, the control plane is decoupled from the data plane and is managed by a logically centralised controller that 


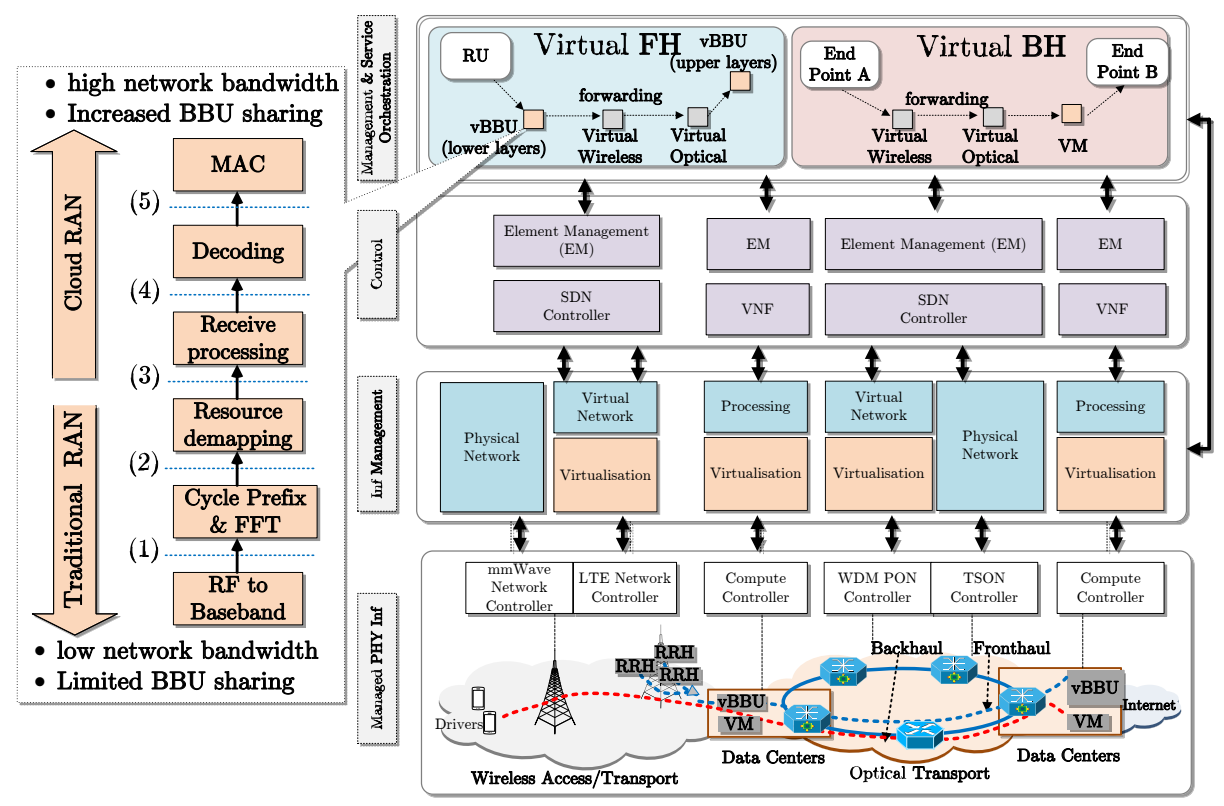

Figure 1. The overarching architecture supporting functional split processing [7].

has a holistic view of the network [8]. At the same time, NFV enables the execution of network functions on commodity hardware (general-purpose servers) by leveraging software virtualisation techniques [8]. Through joint SDN and NFV consideration, significant benefits can be achieved. These are the flexible, dynamic and efficient use of the infrastructure resources, simplification of the infrastructure and its management, increased scalability and sustainability as well as provisioning of orchestrated e2e services.

This layered architecture includes a Physical Infrastructure (PI) layer that comprises a converged optical/wireless transport network able to interconnect RUs, end-users and computing resources. The wireless access part includes dynamically programmable, high capacity, low latency, point-to-multipoint (P2MP) mmWave transceivers, cooperating with Sub-6 GHz systems. The optical transport relies on a hybrid Wavelength Division Multiplexing (WDM) passive optical network (PON) and active optical network solutions able to handle elastic sub-wavelength switching granularities.

The PI will be managed by the Infrastructure Management layer (IML) responsible for the management of the different technology domains and the creation of virtual infrastructure and PI slices comprising heterogeneous resources. These infrastructure slices will enable virtual operator models providing both $\mathrm{FH}$ and $\mathrm{BH}$ services. The IML will communicate with the various network and compute controllers that are responsible for retrieving information and communicating with the individual domains. Once the information has been collected, the resources are abstracted and virtualised. From the architectural and functional perspective, the infrastructure management layer

Trans. Emerging Tel. Tech. 2016; 00:1-8 (C) 2016 John Wiley \& Sons, Ltd. DOI: $10.1002 /$ ett

Prepared using ettauth.cls addresses all virtualisation associated functions as well as the virtual resource management functions. Management of traditional non-virtualised PIs can be also supported.

Cross-domain orchestration of the virtual and physical infrastructures, created and exposed by the IML to the higher layers, will be carried out by a control layer. This layer needs to implement converged control and management procedures for dynamic and automated provisioning of e2e connectivity services according to specific QoS considerations.

Finally, a Management and Service Orchestration layer responsible for the converged orchestration of end user services is needed. This can be used for the composition and delivery of multi-tenant chains of virtualised network functions. In addition, it can perform resource orchestration through NFV resources across multiple Virtual Infrastructure Managers (VIMs) and include lifecycle management of Network Services, supporting Network Service Orchestration functions. Service Orchestration layer related activities are out of scope of the $5 \mathrm{G}-\mathrm{XHaul}$ project.

\section{5G-XHAUL DEVELOPMENT FOCUS}

5G-XHaul is defining a converged wireless and optical transport network offering a converged datapath to an SDN-based control plane.

\subsection{G-XHaul Data Plane Overview}

One of the first steps to understand the nature of the envisioned $5 \mathrm{G}$ transport network has been so far to work on analysing the diverse functional splits from the RAN perspective. This means identifying the optimal ways to 
split a RAN processing chain in a Remote Unit (RU) and a Centralised Unit (CU), and studying the trade-offs of placing these RUs/CUs in distributed or centralised locations [9]. The 5G-XHaul transport network consists of an heterogeneous data plane involving advanced wireless and optical network technologies and designed for coping with the requirements imposed by the RAN.

\subsubsection{Functional splits and Transport Classes}

As mentioned in the introduction, new 5G RAN technologies such as mmWave or Massive MIMO will greatly increase the requirements on FH networks. However, a full centralisation of baseband processing might not be beneficial in all scenarios or for all air interface technologies. Hence, different functional splits (i.e., the allocation of baseband functionality to either RUs or CUs [9][10]) could be employed in 5G networks to enable a high degree of centralisation where it promises benefits. In other scenarios, to reduce costs, the transport network could have much less stringent requirements. These functional splits blur the difference between traditional $\mathrm{FH}$ and $\mathrm{BH}$ network, which motivates one key concept of 5G-XHaul, the converged transport network. The converged transport network unifies the FH and $\mathrm{BH}$ equipment and, hence, reduce deployment as well as operational and maintenance cost. As each of the functional splits imposes a different set of requirements on the transport network, 5G-XHaul has introduced the notion of transport classes (TCs) [11][12]. These TCs would be used to identify traffic corresponding to different sets of requirements in order to facilitate, e.g., a prioritisation of processing with a low overhead in the transport network.

5G-XHaul focuses on three functional splits, illustrated in Figure 2. Split A basically corresponds to a full centralisation as utilised by the CPRI standard, which is used in most of today's C-RAN deployments. The only difference to CPRI is that antenna mapping is performed decentralised, to enable large antenna arrays for beamforming (BF) without having to transport a separate stream for each antenna element. Otherwise, all processing is centralised and I/Q samples are forwarded between CU and RU. However, this comes at the price of the aforementioned stringent requirements in terms of data rate, latency, and synchronisation. One dominant disadvantage of CPRI is that the FH data rate is constant and independent of the actual load of the cell.

Split B utilises decentralised resource mapping. Thereby, most PHY processing can still be performed centrally, but only actually utilised resource blocks need to be forwarded on the transport network. This can greatly reduce the capacity to be deployed in the aggregation segment of transport networks [11][12], and is mostly in line with the Next Generation Fronthaul Interface (NGFI) currently under investigation [13][14].

Splits A and B require synchronisation in order to align samples, hence a transport class was introduced for synchronisation services as mentioned above. As the most stringent delay requirement in CPRI arises from

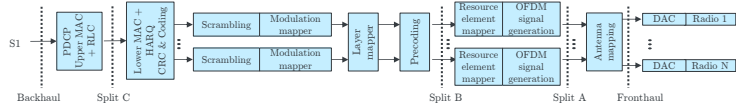

Figure 2. Functional splits options investigated by 5G-XHaul.

the hybrid automated repeat request (HARQ) scheme, split $\mathrm{C}$ decentralises all processing up to and including HARQ. Thereby, the HARQ delay requirement for the FH network is removed, allowing for greater distances between RUs and CUs or allowing for additional latency contributions by transport nodes such as routers or switches. However, this disables the option for centralised PHY-layer processing, and hence centralisation gains can only come from technologies such as centralised scheduling or overall network management. In terms of overall transport requirements this split is similar to traditional BH. As a final remark, it is worth noting that a particular split is better suited for a certain scenario than for all, but it is expected that multiple splits will coexist within the same $5 \mathrm{G}$ network [11].

As an output of this analysis, $5 \mathrm{G}-\mathrm{XHaul}$ is proposing a set of TCs as a foundation of $5 \mathrm{G}$ transport. These TCs have been identified for: a) TC0: synchronisation traffic, b) TC1: transport of I/Q samples/tactile internet traffic, c) TC2: packet-based FH with statistical multiplexing, and d) TC3: traditional $\mathrm{BH}$ services.

\subsubsection{Heterogeneous Technologies}

In order to fulfil the requirements associated to the TCs introduced in Section 3.1.1, 5G-XHaul focuses on developing novel programmability features on data plane technologies. For example: 1) Flexibly allocating time and frequency resources in high capacity, flexible optical transport, 2) Dynamically turning off WDM-PON Optical Network Unit (ONU) transceivers to save energy, or 3) Reconfiguring beam directions in mmWave nodes to enable P2MP in the Small Cell wireless BH. Some of these enabling technologies are briefly introduced below.

Optical Technologies: TSON, WDM-PON 5G optical transport networks able to support converged $\mathrm{FH}$ and $\mathrm{BH}$ services need to provide a high degree of flexibility both in time and frequency domains. Adaptability, scalability, energy efficiency and resilience are other requirements of key importance.

A key enabler supporting the feasibility of this approach is the adoption of a high capacity, flexible optical transport comprising a combination of passive and active solutions. PON solutions will be based on WDM-PONs, while active solutions will adopt more flexible and dynamic WDM technologies such as the Time-Shared Optical Network (TSON).

A pictorial representation of the 5G-XHaul optical transport is shown in Figure 3. TSON has already been deployed at a European level network testbed to efficiently and effectively demonstrate advanced backhauling capabilities [15]. It is designed and implemented as a novel 


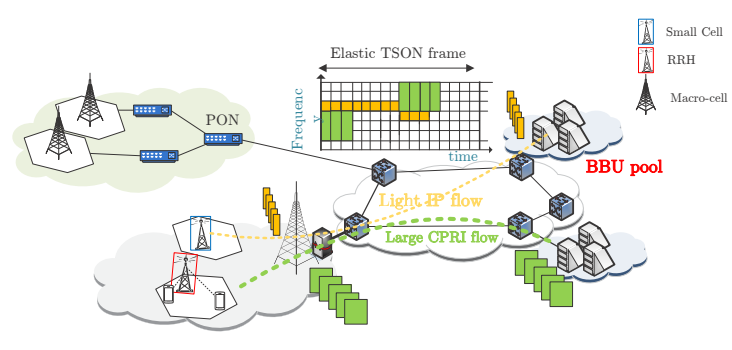

Figure 3. 5G-XHaul Optical Transport

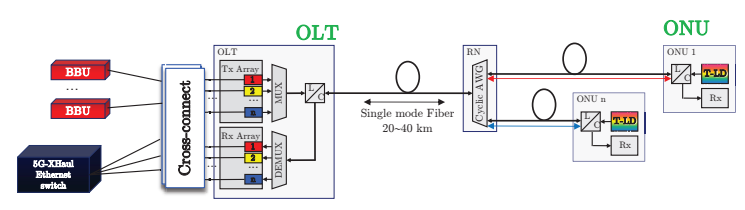

Figure 4. 5G-XHaul WDM-PON Solution.

frame-based, time multiplexing metro network solution, offering dynamic connectivity with fine bandwidth granularity [16].

The TSON framework offers a very flexible optical platform that supports sub-wavelength switching of $10 \mathrm{G}$ Ethernet (10GE) frames, flexible length, programmable frames varying from $64 \mathrm{~ns}$ to $25.6 \mathrm{us}$ and variable bit rates spanning from of $30 \mathrm{Mbps}$ up to $6 \mathrm{Gbps}$. As TSON's operational characteristics can be dynamically modified, varying service related requirements can be supported. Parameters that can be dynamically modified in the current TSON version include bit-rate, burst length, connectivity type and QoS. Extensions planned to be developed in the framework of 5G-XHaul will include also elastic bandwidth allocation and more stringent synchronisation capabilities.

In combination with the TSON technology described above, a WDM-PON solution is designed to deliver the point-to-point $(\mathrm{P} 2 \mathrm{P})$ connectivity between regional data centers that host BBUs and the RUs, i.e. the RAN network, and also able to provide $\mathrm{BH}$ connectivity from the BBU to an Ethernet switch, as shown in Figure 4. Each WDM channel is currently able to achieve bit rates up to $10 \mathrm{Gbps}$, over a transmission distance of 20 to $40 \mathrm{~km}$.

A wide variety of flows will be transported over the $5 \mathrm{G}$ XHaul infrastructure, which span from small control flows to medium IP BH flows and large Cloud-RAN FH flows. Therefore, flexible addressing of individual transport flows between different BBUs and ONUs is required in the proposed system. In addition, flexible RAN networks incorporating flexible centralisation strategies also need to be orchestrated by the 5G-XHaul control plane. In order to realise such a dynamicity in the RAN deployment, an electrical or optical cross-connection functionality between the BBU pool and the transceiver array of the WDM-PON system is provided, such that the traffic flow from each BBU can be flexibly assigned to any desired RRH.
Wireless Technologies: mmWave, Sub-6 GHz Optical fibre links are widely available in urban environments but still their deployment is not fast enough to match the sheer number of small cells required to serve the upcoming demand in mobile data. The high deployment cost of optical fibre will be alleviated in 5G-XHaul with the use of mmWave communications for $\mathrm{BH}$ purposes. The goal is to deliver high capacities - provide the potential gigabit-per-second traffic demand for wireless transport - using mmWave links operating in the $60 \mathrm{GHz}$ band. In this context, it is important to exploit novel radio signal processing methods in the PHY layer, demonstrating that Line-of-Sight and Non-Line-of-Sight performance for mmWave systems can be enhanced.

In very dense RANs, multi-hop wireless topologies for the $\mathrm{BH}$ are required where wireless links will transport the aggregated traffic of several BSs. The idea is to overcome the limitation imposed by static P2P links with dynamic P2MP mmWave connectivity models. 5G-XHaul will develop BF chipsets and design techniques to allow dynamic and autonomic reconfiguration of the mmWave $\mathrm{BH}$, enabling the network management to control the $\mathrm{BF}$ weights used at individual mmWave nodes. These techniques will also allow the implementation of different P2MP schemes over several mmWave nodes.

Antennas for P2MP wireless $\mathrm{BH}$ at $60 \mathrm{GHz}$ have to be highly directive, with high gain enabling efficient $\mathrm{BF}$ and easy integration with the BF chipset. Planar (printed) antenna arrays are considered due to their small form factor, good reproducibility and low cost. Phased antenna arrays with patches or dipoles as basic antenna elements are investigated, with the target to enable scanning in both horizontal and vertical direction. The advantages and limitations of both types are studied and the one with better properties (gain and complexity) will be chosen for implementation.

Sub-6 GHz technologies, such as IEEE 802.11ac, will be considered as one of the key building blocks of the $5 \mathrm{G}$ XHaul wireless BH. The network-wide performance of the Sub-6 GHz BH will be improved through the intelligent orchestration of the individual BH elements. In 5G-XHaul, the Sub-6 GHz wireless BH will be tightly coupled with the mmWave $\mathrm{BH}$ in order to implement an intelligent transport network. For example, traditional IP BH flows could be transported through the Sub- $6 \mathrm{GHz} \mathrm{BH}$ while the mmWave $\mathrm{BH}$ is devoted to transport heavy CloudRAN FH flows. Indeed, 5G-XHaul will consider deeper levels of integration where the Sub- $6 \mathrm{GHz} \mathrm{BH}$ will be used to transport control plane information to configure the mmWave transport. It is worth mentioning that Sub$1 \mathrm{GHz}$ transmissions can also be integrated as part of the $5 \mathrm{G}-\mathrm{XHaul}$ portfolio where bandwidth requirements are relaxed. Sub-1 GHz technologies, such as the new IEEE 802.11ah, provide longer and more reliable wireless links (up to 10x the range of IEEE 802.11ac), at the cost of reducing capacity. 


\subsection{G-XHaul Control Plane Overview}

The 5G-XHaul control plane allows tenants - users of the 5G-XHaul transport system -, which receive connectivity services over the transport network as, e.g. virtual $5 \mathrm{G}$ network operators - to define a transport slice specifying how a set of distributed physical or tenantspecific virtual functions are connected through the $5 \mathrm{G}-$ XHaul infrastructure. In addition, the 5G-XHaul control plane features a North Bound Interface (NBI) that allows tenants to independently control the functions in their transport slices.

In order to support concurrent per-tenant slices in an efficient and scalable way, 5G-XHaul decouples the per-tenant state from the transport-specific state. Following architectural approaches that have been proven to scale in data center environments [17], 5G-XHaul keeps the per-slice state in an overlay composed of edge devices, known as Edge Transport Nodes (ETNs), and illustrated in Figure 5. Consequently, the underlay transport infrastructure focuses on providing connectivity between ETNs in a slice agnostic manner. This approach does not only allow 5G-XHaul to scale, but it also allows to integrate diverse transport technologies in the transport underlay, which is key to enable an easy migration path from legacy transport networks to a 5G-XHaul transport.

However, although being able to function with legacy transport underlays, 5G-XHaul features native support for a transport underlay based on an Ethernet-like datapath and an SDN control plane. Specifically, to improve scalability and support for heterogeneous technologies, 5G-XHaul partitions the transport infrastructure in a set of control plane areas, depicted as Tier-0 Areas (T0-Area) in Figure 5, where transport devices within each area are controlled by a corresponding Tier- 0 Controller (T0-Ctrlr). Notice in Figure 5 how a T0-Area consists of ETNs that connect per-tenant functions to the 5G-XHaul infrastructure, Inter-Area Transport Nodes (IATNs), which interconnect T0-Areas, and Transport Nodes (TNs) connecting ETNs and IATNs within an area. ETNs, TNs and IATNs are controlled by the corresponding T0-Ctrlr using specific southbound interfaces (SBIs). The control plane is organised hierarchically, whereby T0 controllers feature a NBI towards Tier 1 controllers (T1Ctrlr), which operate at a higher abstraction level, and, among other functions, support forwarding at the inter-area level. At the highest layer of the hierarchy, we can find the top level controller offering the 5G-XHaul NBI to services and other components of the $5 \mathrm{G}$ system.

Edge Transport Nodes (ETNs) ETNs maintain perslice state providing $5 \mathrm{G}-\mathrm{XHaul}$ tenants the required abstraction to operate on their slices. For this purpose, an ETN features the internal components shown in Figure 6. On the control plane, an ETN instantiates a logical datapath for each tenant having functions connected to that ETN. Logical datapaths receive high level control policies from the tenant's own control plane, and push those policies to a local SDN controller in the ETN. The

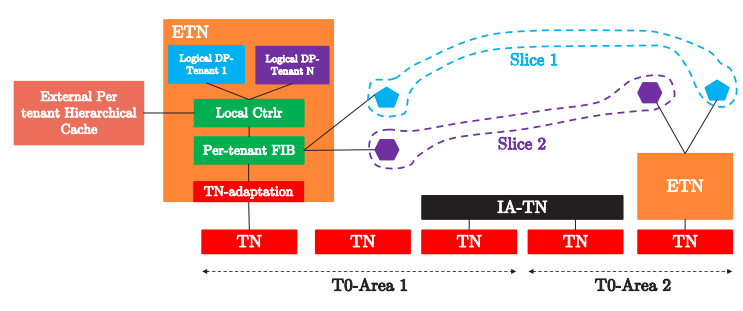

Figure 6. Detailed view of an ETN.

local controller in the ETN implements the corresponding per-tenant state by accordingly placing the rules on each logical datapath. Introducing multi-tenancy support at this last controller layer, which runs on the actual forwarding element, leaves intact the underlying performance-centric multi-stage FIB architecture design that performs the actual packet forwarding. Hence, a critical aspect in the design of the ETN is the datapath delay introduced by the per-tenant Forwarding Information Base (FIB). In order to minimise this delay, tenant-specific rules are often evicted, resulting in a small number of simultaneous rules hosted in the FIB. Consequently, a hierarchical structure of rule caches is used to scale up to a large number of rules coming from different tenants. Finally, the per-tenant FIB matches tenant-specific rules and inserts packets into transportspecific tunnels. A Transport Network adaptation function is included in the ETN that pushes the corresponding transport header before injecting the packets into the transport network. In the case of a native 5G-XHaul transport, the TN Adaptation function injects an outer Ethernet header like the one used in Provider Backbone Bridging (PBB) [18].

Transport Nodes (TNs) TNs provide connectivity between ETNs and IATNs within a given T0-Area by means of transport tunnels. Thus, TNs forward incoming packets based on the corresponding tunnel identifiers, which, in the case of the native 5G-XHaul transport underlay, are defined based on a destination MAC address, a transport specific VLAN, and the I-SID field identifying a transport slice. The proposed Ethernet-based datapath is designed to support a variety of forwarding paradigms such as pinned down tunnels and source routing [19].

Inter Area Transport Nodes (IATNs) IATNs connect together multiple T0-Areas, and, as depicted in Figure 6, are functionally composed of a set of TNs, one for each of the areas connected by that IATN, joined together by an IATN specific control and data plane functions. In the control plane, the IATN function conveys to the 5G-XHaul control plane the T0-Areas that can be reached through this IATN. In the data plane, the IATN translates transport tunnel headers when adjacent areas connected by the IATN feature different transport technologies.

\subsubsection{G-XHaul QoS and TE framework}

5G-XHaul considers a two level QoS framework. At the underlay transport level, the four core transport classes 


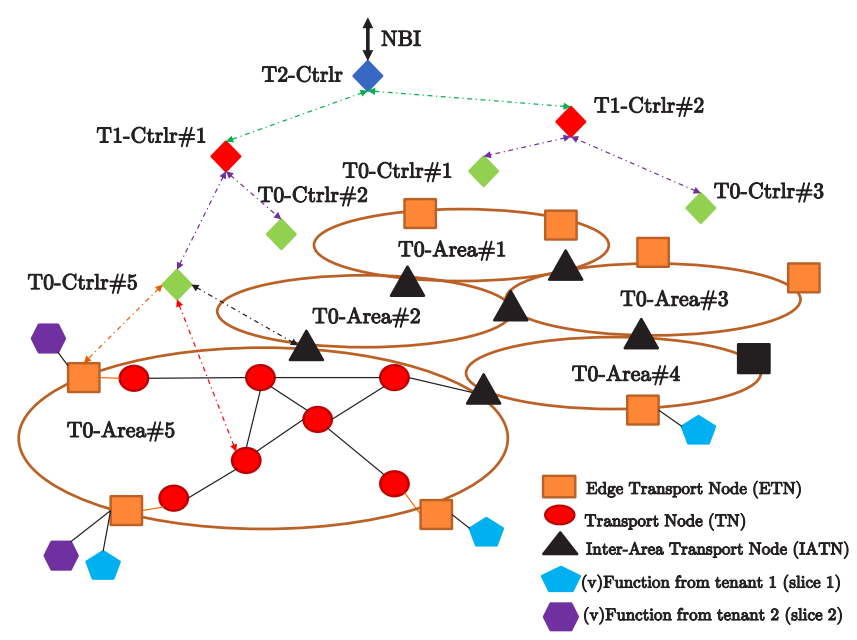

Figure 5. 5G-XHaul Control Plane architecture.

introduced in Section 3, are supported between ETNs and IATNs within each area through the instantiation of different transport tunnels for each class, which, for example, allows to consider minimum delay paths for TC0 and TC1. In addition, within an ETN, and possibly all nodes even including TNs, QoS functionalities are included such as Priority or Weighted fair queueing (WFQ) schedulers that allow to prioritise traffic injected at each transport tunnel interface at the inter-slice and intra-slice level.

Traffic Engineering (TE) functions at different timescales are also supported by the hierarchical 5GXHaul control plane. Local and T0 controllers make more dynamic TE decisions at short timescales while higher tier controllers implement TE functions at longer epochs. Examples of TE functions supported include P2P and P2MP tunnel setup, load balancing between alternative (equal cost) transport tunnels, resilience through the establishment of backup tunnels, traffic policing and shaping.

\subsubsection{G-XHaul RAN-Transport Interface}

A core function of the $5 \mathrm{G}-\mathrm{XHaul}$ control plane is the ability to interface with $4 \mathrm{G}$ or $5 \mathrm{G}$ RANs in order to estimate spatio-temporal demand variations in the RAN and allocate transport resources accordingly. For this purpose, 5G-XHaul is developing several interfaces that allow the exchange of information between transport and RAN at different time scales. First, an interface is considered between the upper tiers of the 5G-XHaul control plane and the Mobility Management Entity - or its $5 \mathrm{G}$ equivalent - of the Mobile Network in order to monitor varying traffic demands at a macro scale (e.g. by means of anonymised traces, such as number of users per tracking area, or estimated weekly patterns), which aid planning decisions in the transport network. Second, an interface between a RAN controller and the T0 controller is considered at shorter time scales that can be used to optimise RAN transport coordination for handover or load balancing purposes. Finally, a tightly coupled interface between the RAN scheduler and the 5G-XHaul ETN is considered to allow the RAN scheduler to quickly change the point of attachment of a flow, which is a critical feature in cell-less architectures considered, e.g. in mmWave RANs [20].

\section{5G-XHAUL CONTRIBUTION TO STANDARDS}

5G-XHaul is well timed to help shape the transport network definition within 5G standardisation. Early results can be fed into the study phase of $5 \mathrm{G}$ definition, which is currently underway in 3GPP, whilst partners can push later findings into the normative specifications. The target date for the first standards release by 3GPP is late 2020 . At the same time, work is ongoing to specify transport technologies that are not necessarily targeted solely at $5 \mathrm{G}$, for example in optical and microwave $\mathrm{FH} / \mathrm{BH}$, so the project aims to contribute here also.

This project has identified a number of domains and standardisation bodies therein where contributions can be made. The functional architecture of the 5G RAN and CN together with interfaces will be defined in 3GPP, guided by NGMN and approved by the ITU-T. The sharing of transport links for $\mathrm{FH}$ and $\mathrm{BH}$, and the sharing of radio links for access and $\mathrm{BH}$, are examples of aspects where the project can contribute. As described above, the project will develop and demonstrate both wireless and optical transport technologies, with outputs feeding into European spectrum regulation, the latest IEEE 802.11 wireless access (ay and ax), and WDM-PON developments in ITUT SG15 and FSAN. Finally, the 5G-XHaul control plane exploits SDN, consequently OpenFlow extensions will be submitted to the ONF, and extensions developed for the OpenDayLight SDN controller used in the demonstration will be fed back to the community. 


\section{CONCLUSIONS}

5G infrastructures will not rely on a single RAT, but will support heterogeneous technologies, including the traditional $2 \mathrm{G}, 3 \mathrm{G}, 4 \mathrm{G}$, as well as new $5 \mathrm{G}$ air interfaces, WiFi solutions, etc. This variety of RATs and the targeted applications have very diverse QoS requirements, need to support different traffic models and rely on different architectures. This paper provides the overall view of the 5G-XHaul transport network architecture, which is based on the convergence of optical and wireless technologies for serving future $5 \mathrm{G}$ RANs. This convergence also applies to $\mathrm{FH}$ and $\mathrm{BH}$ networks. The main goal of a unified convergent transport network is the reduction of cost. This can be achieved by using the same infrastructure to transport data from different functional splits and of different RATs. The network allows dynamic adaption to different traffic patterns and usage scenarios. A coherent management is achieved by SDN functions in conjunction with NFV.

\section{ACKNOWLEDGEMENT}

This work has received funding from the European Union's Horizon 2020 research and innovation programme under grant agreement No. 671551. The European Union and its agencies are not liable or otherwise responsible for the contents of this document; its content reflects the view of its authors only.

\section{REFERENCES}

1. 5G Infrastructure PPP Association, 5G Vision - The 5G Infrastructure Public Private Partnership: the next generation of communication networks and services, White Paper, February 2015.

2. NGMN Alliance, NGMN 5G White Paper, White paper, Feb. 2015. [Online]. Available: http:// ngmn.org/uploads/media/NGMN_5G_ White_Paper_V1_0.pdf. Accessed Mar. 20, 2016.

3. METIS II D1.1 Refined scenarios and requirements, consolidated use cases, and qualitative technoeconomic feasibility assessment, https:// metis-ii.5g-ppp.eu/wp-content/ uploads/METISII_D1.1_v1.0.pdf. Accessed Mar. 30, 2016.

4. Network Functions Virtualisation, ETSI, http:// www.etsi.org/technologies-clusters/ technologies/nfv. Accessed Mar. 23, 2016.

5. ONF, SDN reference architecture, https://www. opennetworking.org/. Accessed Mar. 28, 2016.

6. ETSI GS NFV-SWA 001 V1.1.1 (2014-12), Network Functions Virtualisation (NFV); Virtual Network Functions Architecture, 2014.

7. A. Tzanakaki et al., "5G Infrastructures Supporting End-User and Operational Services: The 5G-XHaul
Architectural Perspective", Accepted to IEEE ICC 2016, Workshop on $5 G$ Architecture, Kuala Lumpur, Malaysia, May 2016.

8. B. Han, V. Gopalakrishnan, L. Ji, S. Lee, ”Network function virtualization: Challenges and opportunities for innovations," IEEE Commun. Mag., vol.53, no.2, pp.90-97, Feb. 2015.

9. J. Bartelt et al., "Fronthaul and backhaul Requirements for Flexibly Centralized Radio Access Net-works", IEEE Wireless Comm. Mag., Vol. 22, no. 5, pp 105111, October 2015.

10. U. Dötsch, M. Doll, H. P. Mayer, F. Schaich, J. Segel, and P. Sehier, "Quantitative Analysis of Split Base Station Processing and Determination of Advantageous Architectures for LTE," Bell Labs Tech. J., vol. 18, no. 1, pp. 105-128, May 2013.

11. J. Bartelt et al., "The Impact of 5G Radio Access Technologies on a Converged Fronthaul and Backhaul Network," submitted to IEEE Wireless Comm., Feb. 2016.

12. Deliverable D2.1 "Requirements Specification and KPIs", 5G-XHaul Project, March 2016.

13. C. L. I, J. Huang, Y. Yuan, S. Ma and R. Duan, "NGFI, the xHaul," 2015 IEEE Globecom Workshops (GC Wkshps), San Diego, CA, 2015, pp. 1-6.

14. China Mobile NGFI White paper: China Mobile, ALU, Nokia, ZTE, Broadcom, Intel, White Paper of NGFI (Next Generation Fronthaul Interface) Version 1.0, October 4, 2015.

15. B. Rahimzadeh Rofoee, K. Katsalis, Y. Yan, Y. Shu, T. Korakis, L. Tassiulas, A. Tzanakaki, G. Zervas, D. Simeonidou, "Demonstration of Servicedifferentiated Communications over Converged Optical Sub-Wavelength and LTE/WiFi Networks using GEANT Link," in proc. of OFC 2015, Los Angeles, California, USA

16. Y. Yan et al., "High performance and flexible FPGAbased time shared optical network (TSON) metro node," Opt. Exp 21, 5499-5504,2013.

17. T. Koponen et al., "Network virtualization in multitenant datacenters [next-generation carrier ethernet transport technologies, " In 11th USENIX Symposium on Networked Systems Design and Implementation (NSDI 2014) (pp. 203-216).

18. D. Fedyk and D. Allan. "Ethernet data plane evolution for provider networks", IEEE Communications Magazine, vol. 46, no. 3, pp. 84-89, March 2008.

19. C. Filsfils, N. K. Nainar, C. Pignataro, J. C. Cardona, P. Francois, "The Segment Routing Architecture", In IEEE Global Communications Conference (GLOBECOM 2015), San Diego, California, USA (pp. 1-6).

20. R. J. Weiler, M. Peter, W. Keusgen, E. C. Strinati, A. De Domenico, I. Filippini, A. Capone, I. Siaud, A.M. Ulmer-Moll, A. Maltsev and T. Haustein, "Enabling 5G backhaul and access with millimeter-waves", In EuCNC 2014, Bologna, Italy, June 2014 (pp. 1-5). 